FERMILAB-Pub-01/129-T

June 2001

\title{
Chiral Loops and Ghost States in the Quenched Scalar Propagator
}

\author{
W. Bardeen ${ }^{1}$, A. Duncan ${ }^{2}$, E. Eichten ${ }^{1}$, N. Isgur ${ }^{3}$ and H. Thacker ${ }^{4}$ \\ ${ }^{1}$ Fermilab, P.O. Box 500, Batavia, IL 60510 \\ ${ }^{2}$ Dept. of Physics and Astronomy, Univ. of Pittsburgh, Pittsburgh, PA 15260 \\ ${ }^{3}$ Jefferson Lab, 12000 Jefferson Avenue, Newport News, VA 23606 \\ ${ }^{4}$ Dept.of Physics, University of Virginia, Charlottesville, VA 22901
}

\begin{abstract}
The scalar, isovector meson propagator is analyzed in quenched QCD, using the MQA pole-shifting ansatz to study the chiral limit. In addition to the expected short-range exponential falloff characteristic of a heavy scalar meson, the propagator also exhibits a longer-range, negative metric contribution which becomes pronounced for smaller quark masses. We show that this is a quenched chiral loop effect associated with the anomalous structure of the $\eta^{\prime}$ propagator in quenched QCD. Both the time dependence and the quark mass dependence of this effect are well-described by a chiral loop diagram corresponding to an $\eta^{\prime}-\pi$ intermediate state, which is light and effectively of negative norm in the quenched approximation. The relevant parameters of the effective Lagrangian describing the scalar sector of the quenched theory are determined.
\end{abstract}




\section{Introduction}

For the foreseeable future, the quenched approximation will continue to play an important role in the study of lattice QCD for both practical and theoretical reasons. The practical reasons are only too obvious, considering the orders-of-magnitude increase in computer resources required for comparable full QCD simulations. But quenched QCD also provides a very instructive counterpoint to the full theory which can yield useful insight into the effect of quark loops in determining hadron structure. This is particularly true in the lightquark limit, where lattice results can be interpreted theoretically with the aid of an effective field theory corresponding to quenched chiral perturbation theory.

The introduction of quenched chiral perturbation theory by Sharpe, Bernard, and Goltermann [1, 2] established the framework for analyzing the chiral behavior of quenched QCD. This work focused attention on the particular, anomalous role of the quenched $\eta^{\prime}$ propagator. The physical $\eta^{\prime}$ is approximately a flavor singlet and gets most of its mass from the axial anomaly via repeated $q \bar{q}$ annihilation. In the quenched approximation, a single annihilation vertex can appear if both sides of the "hairpin diagram" are attached to valence quark lines, but repeated annihilation cannot take place without the closed quark loops of the unquenched theory. Instead of cancelling the Goldstone pole in the valence quark ("connected") diagram, the single annihilation diagram contributes a term to the $\eta^{\prime}$ propagator which has a double Goldstone pole and an overall sign opposite to that of the valence diagram. Unlike full QCD where $\eta^{\prime}$ loops remain infrared finite in the chiral limit, in the quenched theory, the double Goldstone pole of the hairpin term produces additional quenched chiral loop (QCL) singularities which alter the chiral behavior of the theory. This

QCL effect was first observed in lattice results as a deviation from linear behavior of the squared pion mass as a function of quark mass [3, 何. Recently, the quenched chiral limit was extensively investigated in a high-statistics study at $\beta=5.7[5]$. The problem of excep- 
tional configurations, which had prevented previous studies from probing the smaller quark mass region of $m_{\pi}<350 \mathrm{MeV}$, was resolved by the pole-shifting ansatz of the modified quenched approximation (MQA) [6]. The QCL effect was observed in both the pseudoscalar mass and decay constants at the level expected from a direct study of the $\eta^{\prime}$ hairpin mass insertion and the topological susceptibility. This established several independent and quantitatively consistent determinations of the quenched chiral log coefficient parameter $\delta$ (or, equivalently, of the $\eta^{\prime}$ mass insertion $\left.m_{0}^{2}\right)$.

The spectroscopy of scalar mesons has long been one of the murkier areas of hadron phenomenology. The well-established scalar mesons are typically at masses of a GeV or more, although there have been occasional, fleeting experimental indications of lower mass scalar resonances. In the flavor singlet sector, mixing with glueball states further complicates the intepretation of experimental data. This is clearly an area where lattice QCD calculations can be expected to play a crucial role in the future. High-statistics quenched studies will undoubtedly be an important part of this effort. Naively, we might expect that the anomalous chiral behavior induced by the quenched approximation would be of little importance for scalar-meson spectroscopy, since the meson masses involved are all expected to be quite heavy and not particularly sensitive to the details of chiral extrapolation. However, as we show in this paper, a more careful theoretical analysis combined with a precise numerical study of the scalar, isovector meson propagator at very light quark mass reveals perhaps the most striking quenched chiral loop effect yet observed.

The chiral loop effect we discuss here is exhibited very clearly by comparing the scalar, isovector propagator for the heaviest and lightest quark masses studied. These are shown in Figure 11. For the heaviest quark mass the propagator is a positive and rapidly falling exponential, as expected from heavy scalar meson intermediate states. The fit shown is a sum of two exponentials with the excited state $a_{0}^{*}$ mass extracted by comparing local- 


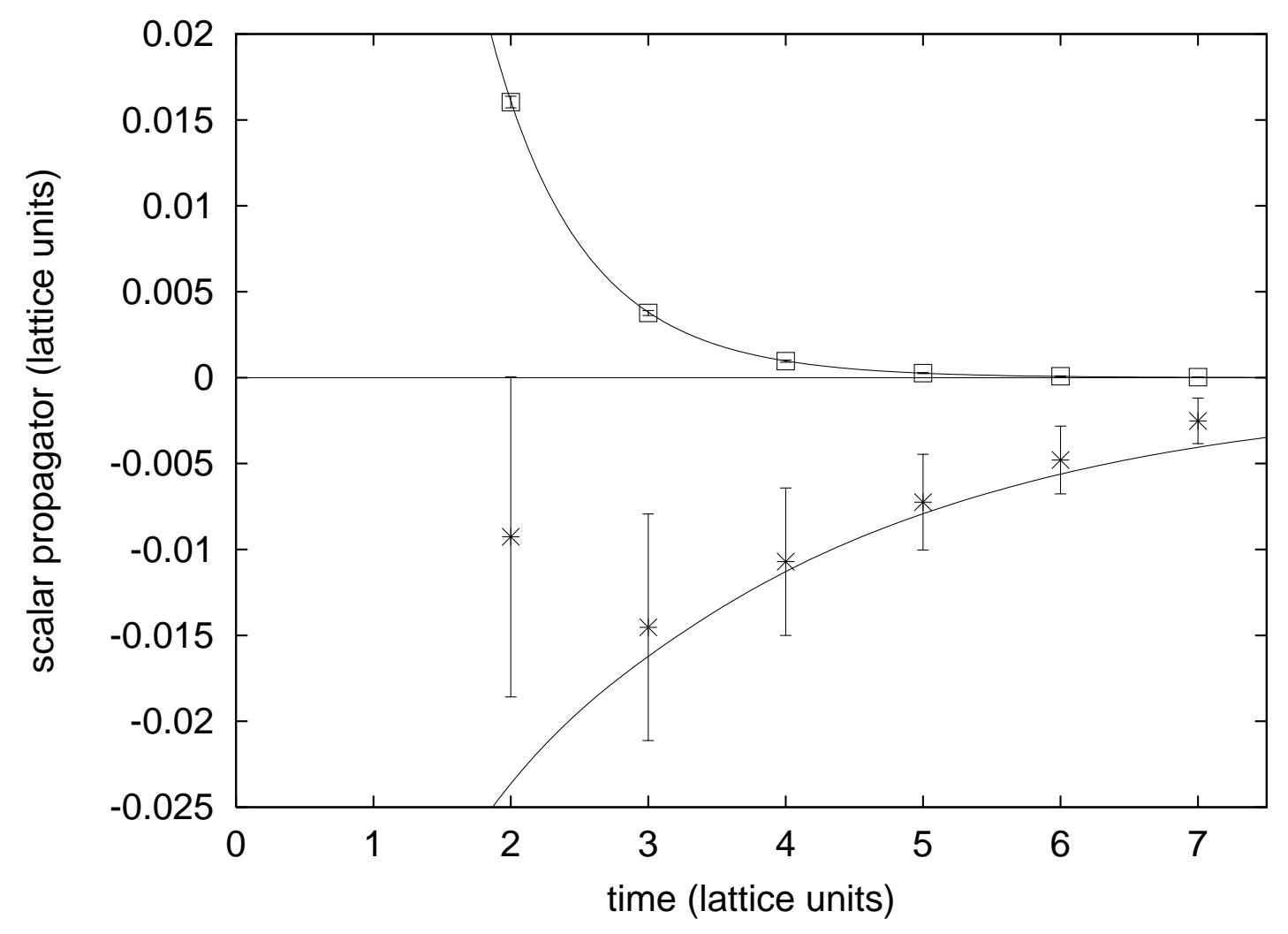

Figure 1: The scalar propagator for the heaviest $(\kappa=.1400)$ and lightest $(\kappa=$ .1428) quark masses studied. For heavy quarks (boxes) the propagator is positive and exponentially falling. The (upper) solid curve is a two-exponential fit. For light quarks $(*$ 's) the propagator exhibits a negative quenched chiral loop effect.

The lower solid curve is the prediction of one-loop chiral perturbation theory for this term. (See Section 2.) 
local, smeared-local, and smeared-smeared correlators. (See discussion in Section 3.) By contrast, the scalar propagator for lighter quarks exhibits a qualitatively different behavior. [The lower curve in Figure 1 is the one-loop quenched $\chi P T$ prediction for the contribution to the scalar propagator of the $\eta^{\prime}$ - $\pi$ intermediate state only. See below and Section 2.] This unusual behavior has two qualitative features which point clearly to a specific theoretical interpretation: (1) The additional component becomes much more prominent for the lightest quark masses, and (2) the sign of this component is negative (i.e. opposite to that required by positivity of the spectral function). Both of these properties suggest that the new component is an effect of the $\eta^{\prime}-\pi$ intermediate state, which is light in the quenched approximation. This contribution arises from the "hairpin + pion" diagram shown in Figure 2(b). This interpretation also explains the fact that this component has a negative spectral weight. The hairpin (single mass insertion) diagram appears in the $\eta^{\prime}$ propagator with the opposite sign from that of the full propagator. In full QCD the $\eta^{\prime}-\pi$ intermediate state would arise from three different types of quark line diagrams, Figure 2(a) with a single vacuum loop going around the entire diagram, Figure 2(b) with a single hairpin vertex, and the graphs of type 2(c) with one or more vacuum bubbles inserted into the $\eta^{\prime}$ propagator. Of these three, only the hairpin diagram, Figure 2(b), is included in the quenched approximation. Since the single hairpin insertion has an overall negative sign (corresponding to a positive $\eta^{\prime}$ mass shift), the sign of this contribution is opposite to that which would be required by spectral positivity. The prominence of this negative term in our measured propagator is a clear example of the unitarity violation induced by the quenched approximation. [In the Bernard-Goltermann scalar ghost quark formulation of quenched chiral perturbation theory [2], the negative sign of the scalar propagator arises from the dominance of a negative metric state consisting of a pair of ghost-quark mesons.] 


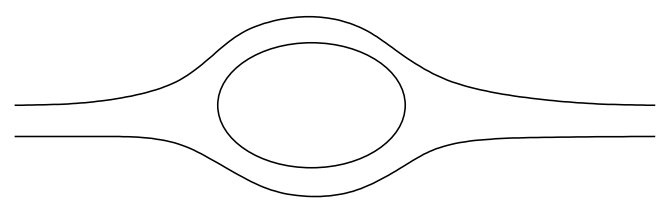

(a)

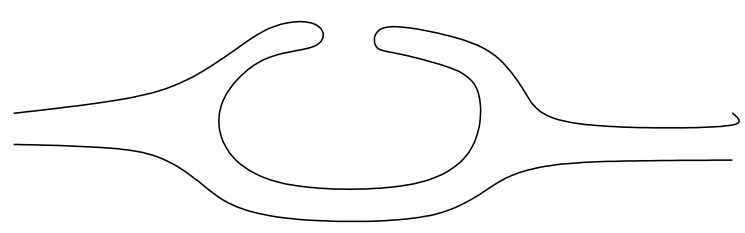

(b)

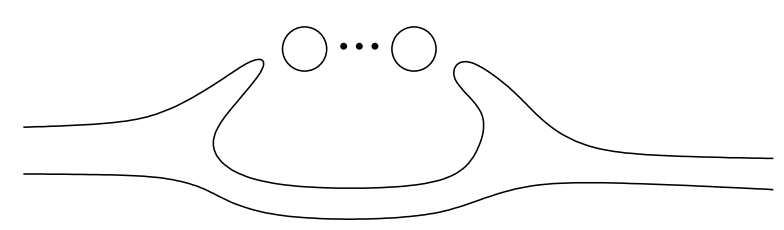

(c)

Figure 2: Quark line diagrams which contribute to the $\eta^{\prime}-\pi$ intermediate state in the scalar isovector propagator. 


\section{Lattice Results for the Scalar Propagator}

In a recent study of quenched chiral logs and the $\eta^{\prime}$ propagator [5], the MQA pole-shifting ansatz was applied to a set of quark propagators for an ensemble of 300 gauge configurations at $\beta=5.7$ on a $12^{3} \times 24$ lattice. The fermion action was clover-improved Wilson-Dirac with

$C_{s w}=1.57$. Nine values of quark mass were used, corresponding to a range of hopping parameters from $\kappa=.1400$ to .1428 . Valence quark propagators were calculated from both local delta-function sources and from exponentially smeared sources in Coulomb gauge. (For further details, see Reference [5].) These pole-shifted quark propagators were used to calculate the scalar meson propagators considered in this paper. The pion masses obtained from this ensemble have values ranging from $m_{\pi} a=0.245$ to 0.603 , and are listed in Table I.

It is natural to expect that the scalar $\bar{\psi} \psi$ correlator in QCD will be dominated by the coupling to the lightest scalar meson which is expected to have a mass larger than $1 \mathrm{GeV}$. For the heaviest quark masses studied here, this is in fact the behavior observed for the quenched propagator. First, consider the scalar propagator for our heaviest quark mass, with $m_{\pi} a=.603$, corresponding to $\kappa=.1400$. (From Reference [5], we have $\kappa_{c}=0.14329$ for clover-improved Wilson-Dirac fermions). The measured values of the local-local, smearedlocal, and smeared-smeared propagators are shown in the log plot in Figure 3. As expected, the plot shows clear evidence of a massive scalar meson. For the smeared-local propagator, the time-dependence is reasonably well described for $t>2$ by an exponential fit with a mass of $M_{s} a=1.25(2)$. The effective mass plot for the smeared-local propagator is shown in Figure 1 for $\kappa=.1400$. For comparison, the effective mass for the local-local and smeared-smeared propagators are also plotted. Although the smearing function used (an exponential with exponent 0.5 in lattice units) was not tuned for this particular problem, it does a good job of removing excited state contamination, giving a reasonably flat effective 


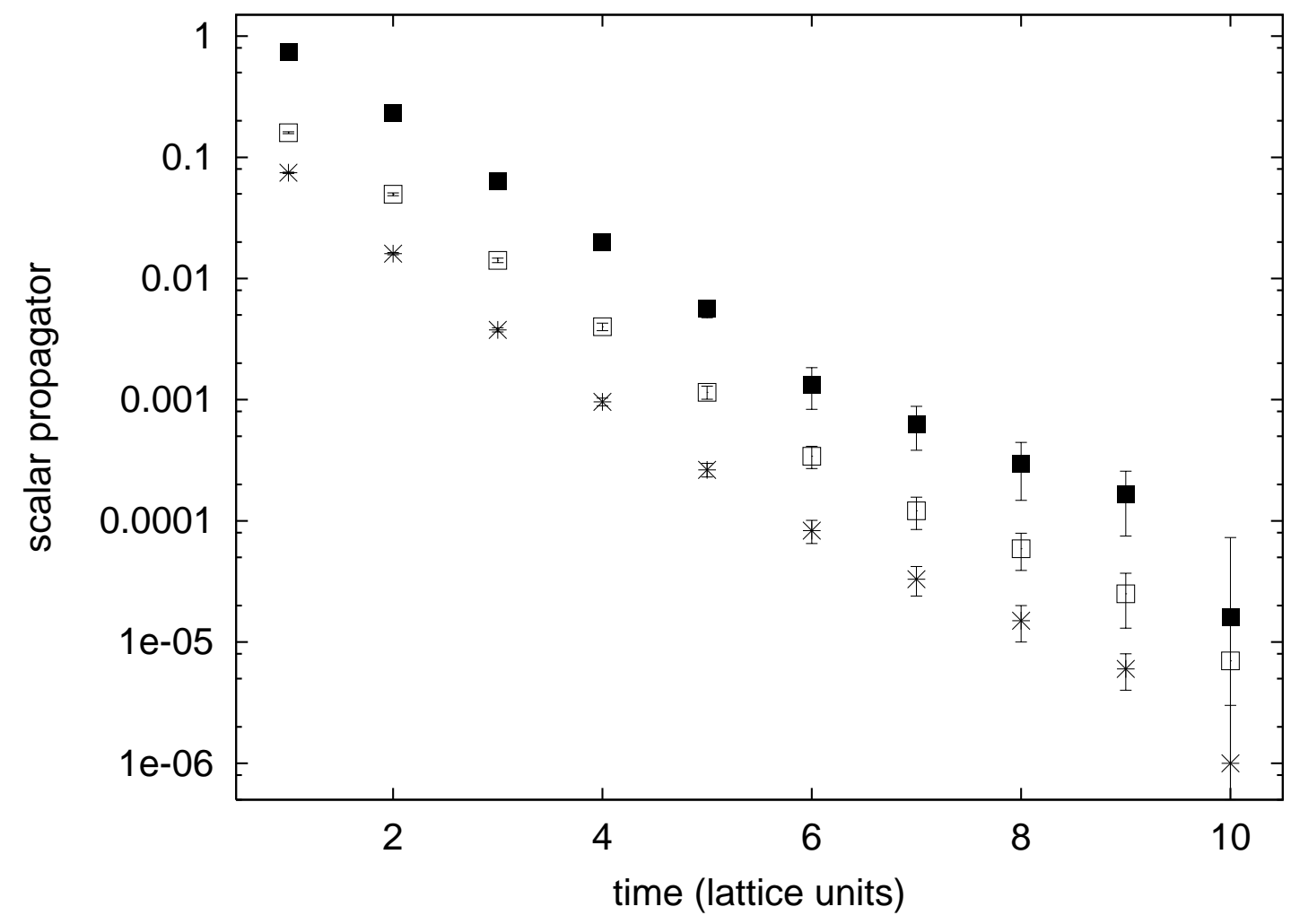

Figure 3: The scalar propagator for the heaviest quark $(\kappa=.1400)$ with local-local $\left({ }^{*}\right.$ 's), smeared-local (empty boxes), and smeared-smeared (full boxes) sources. 


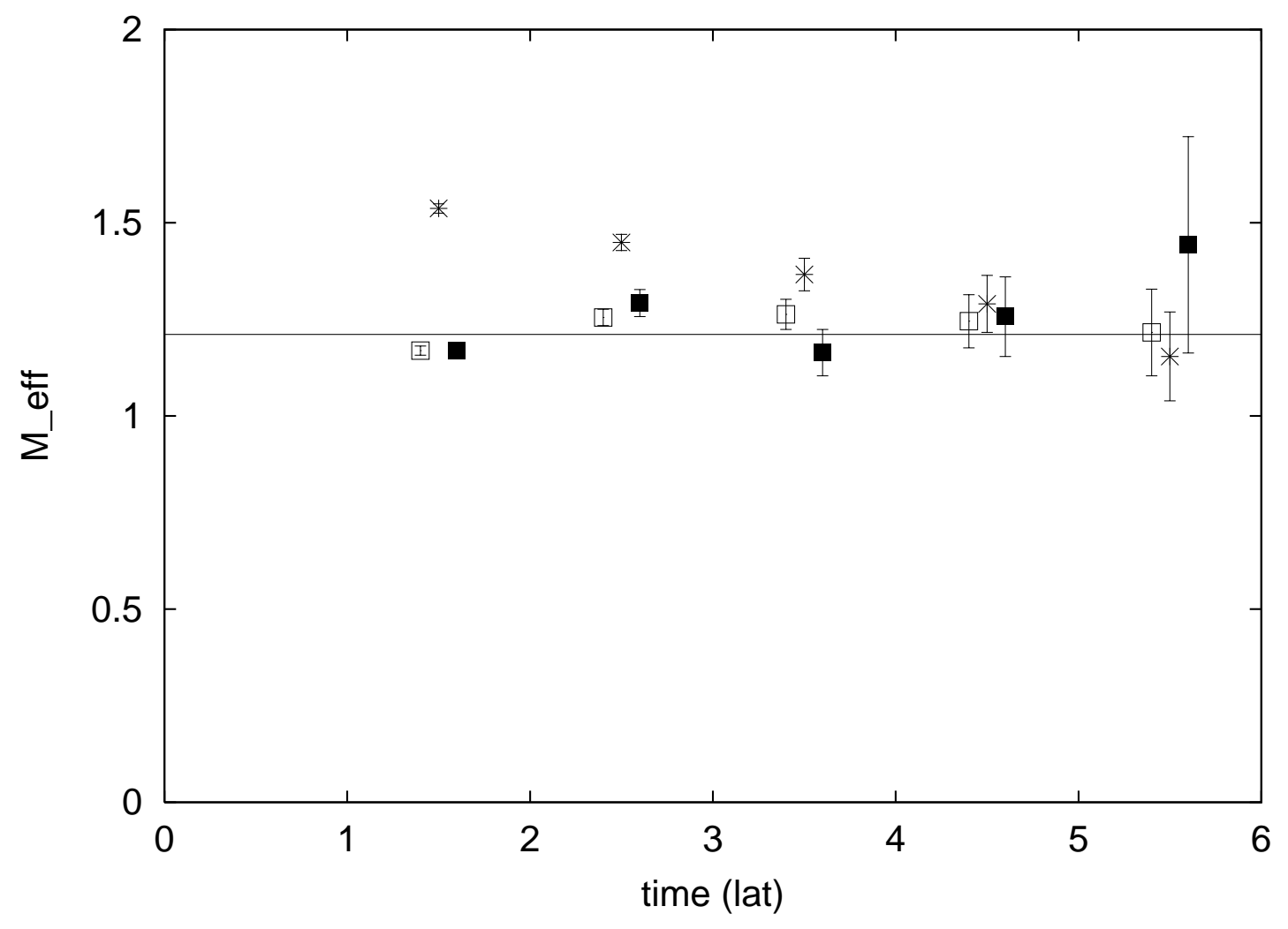

Figure 4: Effective mass plot for $\kappa=.1400$ for local-local (*'s), smeared-local (empty boxes), and smeared-smeared (full boxes) correlators. 


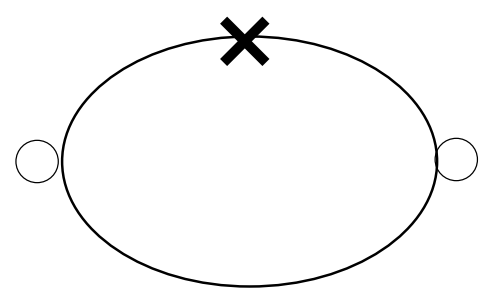

Figure 5: One-loop quenched chiral perturbation theory graph evaluated in Eq. (2). mass over several time slices from $t=2$ to $t=6$, and a nearly identical effective mass plot for smeared-local and smeared-smeared propagators (indicating an absence of excited states in both).

As pointed out in the Introduction, the behavior of the scalar propagator changes dramatically for the lighter quark mass values. The results for the scalar propagator for our lightest quark mass values are shown in Figure 6, with $m_{\pi} a=.245, .267, .307$, and .342 corresponding to $\kappa$ values $(.1428, .1427, .1425$, and .1423$)$ respectively. Here we have plotted the local-local $\bar{\psi} \psi$ correlators. Instead of exhibiting the behavior expected for scalar meson states, these propagators are dominated by a significant negative contribution in the range $t=2$ to $t=7$ which increases for lighter quark masses. We interpret this behavior as a clear signal for the negative metric contribution associated with the $\eta^{\prime}-\pi$ intermediate state discussed in the previous Section.

The $\eta^{\prime}-\pi$ loop interpretation of the anomalous component of the scalar propagator is made more convincing by noting that the leading chiral behavior of this contribution is entirely determined by current algebra in terms of parameters which have already been measured with this ensemble in our previous study of the pseudoscalar propagator [5]. The relevant chiral loop diagram is shown in Figure 5, with the $\times$ denoting the $\eta^{\prime}$-hairpin mass insertion $m_{0}^{2}$, and the o's representing $\bar{\psi} \psi$ operator vertices. If we ignore form factor effects at these vertices, we can calculate the $\eta^{\prime}-\pi$ contribution to the $\bar{\psi} \psi$ propagator in momentum 


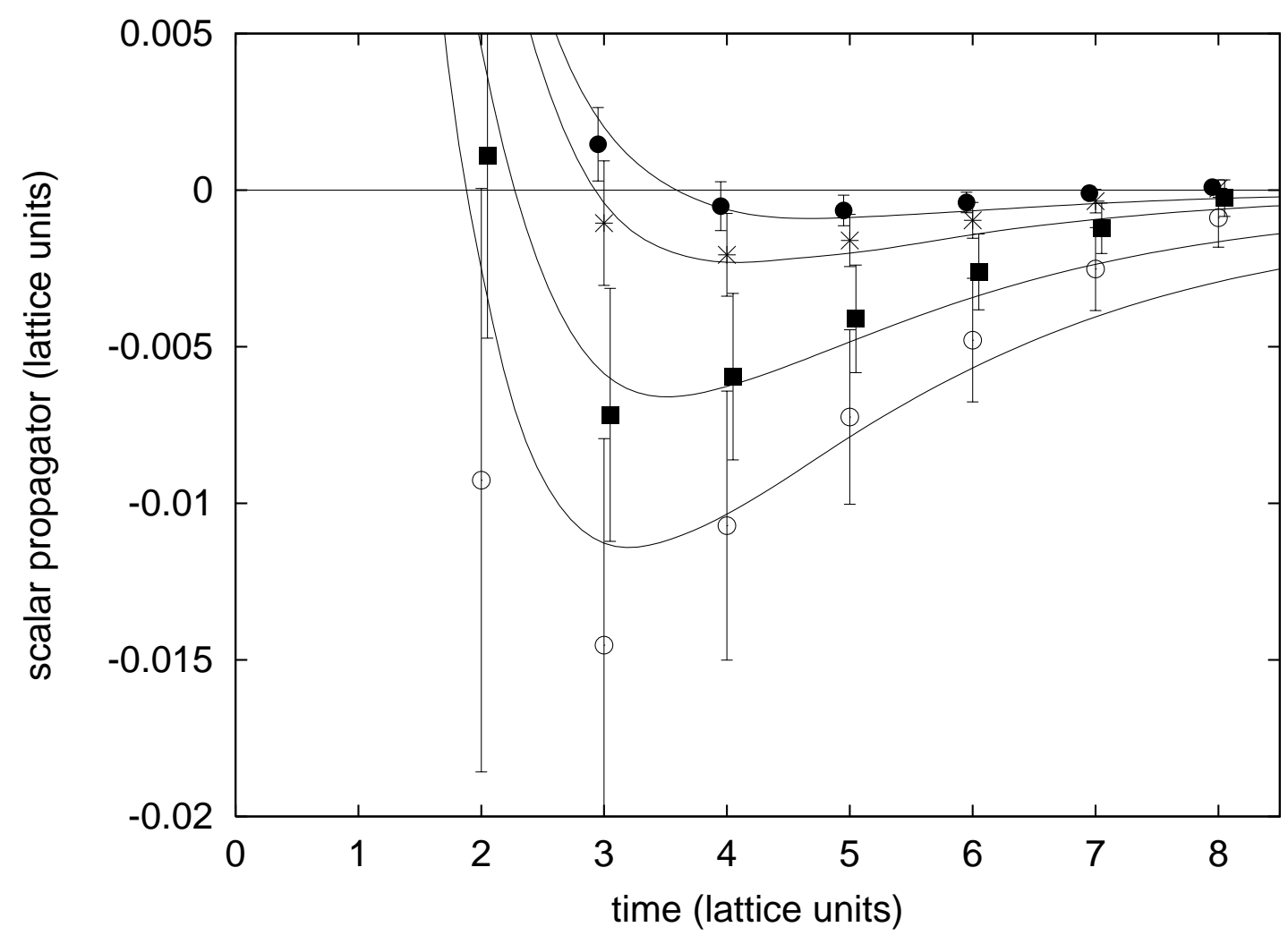

Figure 6: Scalar propagator for $\kappa=.1423(\bullet), .1425(*$ 's), .1427 (solid squares), and .1428 (०). Solid curves are fits to the bubble sum formula, (19). 
space. The propagator

$$
\Delta(p) \equiv \sum_{x} e^{-i p \cdot x}\langle\bar{\psi} \psi(x) \bar{\psi} \psi(0)\rangle
$$

is given in terms of the one-loop integral $B(p)$,

$$
\Delta(p) \sim-4 r_{0}^{2} B(p)
$$

where

$$
B(p)=\frac{1}{V T} \sum_{k} \frac{1}{\left((k+p)^{2}+m_{\pi}^{2}\right)} \frac{m_{0}^{2}}{\left(k^{2}+m_{\pi}^{2}\right)^{2}}
$$

Here, $2 r_{0}$ is the $\bar{\psi} \psi$ to $\eta^{\prime}-\pi$ coupling. (In the next section we will discuss including the scalar meson intermediate state, along with form-factor/unitarization effects). The pion mass values for the hopping parameters used here have already been reported in Reference [5] and are listed here in Table I. The hairpin insertion mass $m_{0}^{2}$ is also determined in Reference [5]. For clover improved fermions with $C_{s w}=1.57$ the value found in the chiral limit (dropping a flavor factor of $\sqrt{3}$ included in Table II of that reference) is

$$
m_{0}=.33(2)
$$

The quark mass dependence of $m_{0}$ was found to be very mild (see Table II of Reference (5)). Here, for simplicity, we will take it to be a mass independent constant given by (4). The only other parameter needed to evaluate the $\eta^{\prime}-\pi$ bubble contribution is the coupling of the scalar $\bar{\psi} \psi$ operator to the $\eta^{\prime}-\pi$ state,

$$
\left\langle 0|\bar{\psi} \psi| \eta^{\prime} \pi\right\rangle
$$

In the quenched theory in the chiral limit, we can relate the threshold value of this matrix element, via a soft- $\eta^{\prime}$ reduction, to the decay constant $f_{P}$, the vacuum-to-one-pion matrix element of the pseudoscalar density. By this argument, we relate the coupling constant in 
(2) to the $m_{\pi}^{2}$ vs. $m_{q}$ slope parameter $r_{0}$ determined in Reference [5],

$$
r_{0}=1.99(12)
$$

Thus, in this approximation the effect of the $\eta^{\prime}-\pi$ bubble, Eq. (2), is completely predicted by chiral symmetry in terms of the constants (4), (6), and the pion masses in Table I. The result for the lightest quark mass $(\kappa=.1428)$ is plotted along with the measured propagator in Figure 1 (lower curve). Considering that the plotted curve has no adjustable parameters, it fits the size and shape of the propagator remarkably well in the time range from $t=3$ to $t=7$. For times $t<3$, the measured propagator deviates from the one-loop expression in the positive direction, indicating that the spectral function also contains a heavy scalar meson. This short-range, positive metric exponential component dominates in the heavier quark cases where the chiral loop effect becomes unimportant. The one-loop result also explains the pion mass dependence of the anomalous propagator component, as shown in Figure 7, where we have plotted the value of the scalar propagator at various time separations as a function of $m_{\pi}^{2}$. The solid curves are the $p_{0}$ Fourier transform at $\vec{p}=0$ of the one-bubble integral (2), with $m_{0}$ and $r_{0}$ given by (四) and (6). Again the agreement with the data is surprisingly good, considering the absence of any adjustable parameters. The accurate description of both the time-dependence and the pion mass dependence leaves little doubt that, for the lightest quark masses, the scalar propagator over the range of times from about $t=3$ to 7 is completely dominated by the $\eta^{\prime}-\pi$ loop. Furthermore, this agreement shows that the soft pion theorem relating the scalar-density matrix element (5) to the corresponding pseudoscalar matrix element is well satisfied by the lattice results. This is somewhat surprising at $\beta=5.7$, where one might have expected substantial chiral symmetry violation from finite lattice-spacing effects.

For short time separations and/or heavier quark masses, an accurate description of the scalar propagator requires positive contributions from scalar meson states along with the 

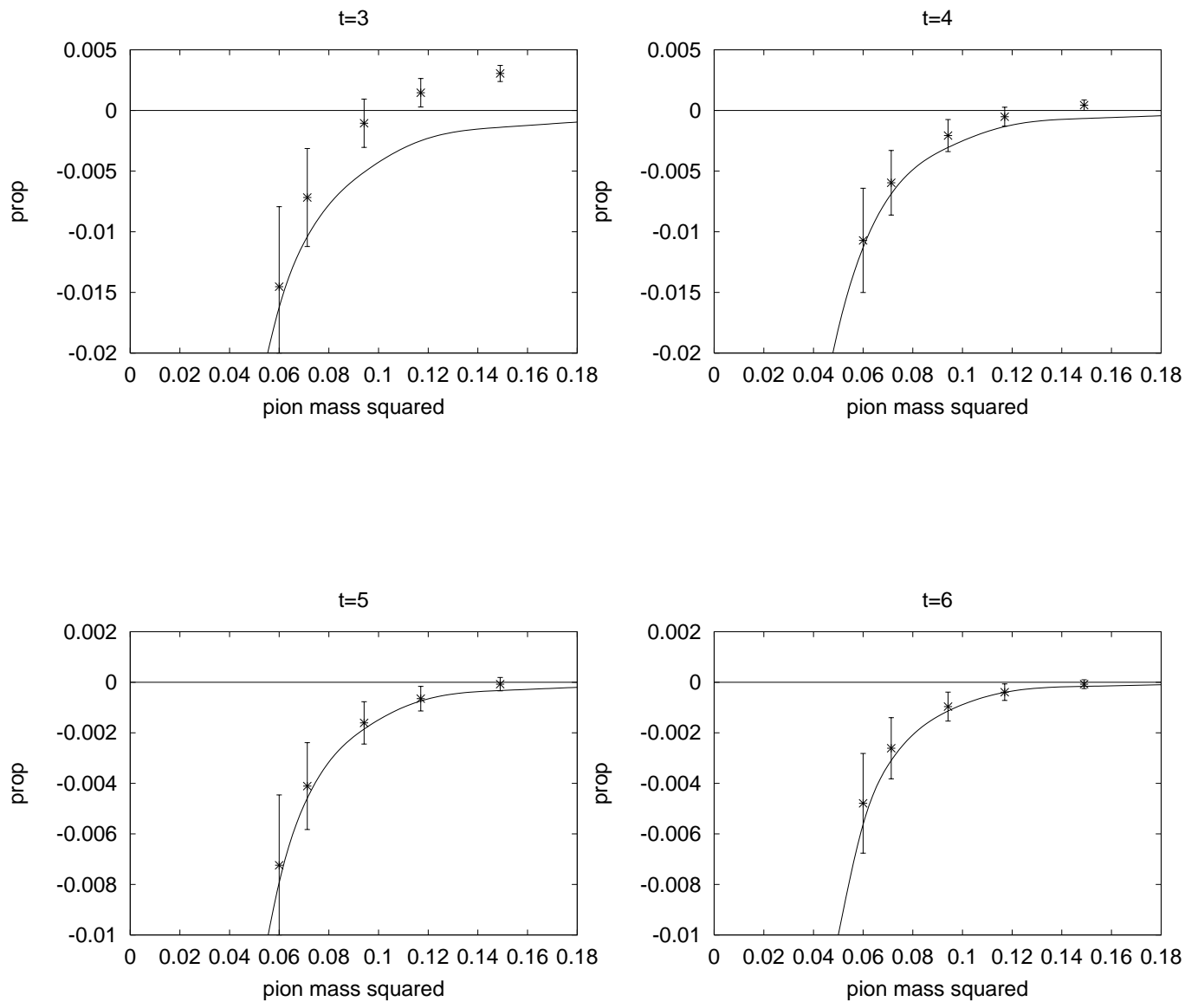

Figure 7: Pion mass dependence of the scalar propagator for $t=3,4,5,6$. 
negative $\eta^{\prime}-\pi$ component. In the next Section, we present a derivation of the quenched scalar propagator based on a detailed chiral Lagrangian description which will be used to fit our data for all quark mass values. The corresponding propagator fits are discussed in Section 4 .

\section{Quenched Chiral Perturbation Theory}

According to the analysis of Bernard and Golterman, low-energy quenched QCD is described by an effective local field theory with degrees of freedom corresponding to meson bound-

states, including not only ordinary $q \bar{q}$ mesons, but also $q^{\prime} \bar{q}, q \bar{q}^{\prime}$, and $q^{\prime} \bar{q}^{\prime}$ mesons, where $q^{\prime}$ is a wrong-statistics ghost quark. Order by order in the $\eta^{\prime}$ mass insertion $m_{0}^{2}$, the ghostquark formalism is easily seen to be equivalent to Sharpe's formulation, which begins with a $U(3) \times U(3)$ chiral Lagrangian combined with rules inferred from the structure of quark-line diagrams in the quenched theory. In this paper, as in our previous analysis 5 we follow the latter approach, invoking a chiral Lagrangian having valence pion and $\eta^{\prime}$ degrees of freedom. To describe quenched QCD, the chiral Lagrangian is supplemented with rules which reflect the suppression of internal quark loops. As a result, all normal pion loops are suppressed except for cactus diagrams involving the $\eta^{\prime}$ meson connected by single insertions of the hairpin mass term. Using this procedure we obtained a consistent fit to our lattice data for the pseudoscalar valence and $\eta^{\prime}$ hairpin propagators and determined the relevant chiral Lagrangian parameters. We now extend this analysis to the isovector, scalar propagator.

The appropriate effective field theory must now include a multiplet of scalar mesons in addition to the pion and $\eta^{\prime}$ degrees of freedom. Using standard chiral Lagrangian methods, the heavy scalar mesons are described by scalar fields transforming nonlinearly under chiral 
symmetry rotations. The resulting chiral Lagrangian is

$$
\begin{aligned}
\mathcal{L} & =\frac{f^{2}}{4} \operatorname{tr}\left\{\partial U \partial U^{\dagger}\right\}+\frac{f^{2}}{4} \operatorname{tr}\left\{\chi^{\dagger} U+U^{\dagger} \chi\right\} \\
& +\frac{1}{4} \operatorname{tr}\{D \sigma D \sigma\}-\frac{1}{4} m_{s}^{2} \operatorname{tr}\{\sigma \sigma\}+g_{s} \operatorname{tr}\left\{\sigma \sqrt{ } U \partial U^{\dagger} \partial U \sqrt{ } U^{\dagger}\right\} \\
& +f_{s} \operatorname{tr}\left\{\chi^{\dagger} \sqrt{ } U \sigma \sqrt{ } U+\chi \sqrt{ } U^{\dagger} \sigma \sqrt{ } U^{\dagger}\right\}+\mathcal{L}_{\text {hairpin }}
\end{aligned}
$$

where $\mathcal{D}$ is a chiral covariant derivative, $m_{s}$ the sigma mass, $g_{s}$ the strength of the chiral invariant coupling of sigma mesons to pions and $f_{s}$ the strength of the corresponding chiral symmetry breaking interactions. Here we denote the scalar field by $\sigma$. (Elsewhere, when referring to particle states, we use the conventional $a_{0}$ and $a_{0}^{*}$ to denote ground-state and excited-state mesons.) The chiral field matrix $U$ contains the pion and $\eta^{\prime}$ degrees of freedom. The $\eta^{\prime}$ mass term is given by the hairpin Lagrangian,

$$
\mathcal{L}_{\text {hairpin }}=-\frac{1}{2} m_{0}^{2}\left(f^{2} / 8\right)\left[i t r \ln \left(U^{\dagger}\right)-i \operatorname{tr} \ln (U)\right]^{2} .
$$

We have not included interactions involving the couplings $L_{5}$ and $L_{8}$ used in our previous analysis of higher order terms in the sigma potential as they will have only small effects on our analysis of the isovector scalar propagator.

Scalar and pseudoscalar quark densities are represented by meson operators in the effective field theory and can be determined from the dependence of the chiral Lagrangian on the spurion field $\chi$. Hence the isovector scalar density operator is given by

$$
\begin{aligned}
\overline{\Psi_{2}} \Psi_{1}= & -\frac{1}{2} r_{0} f^{2}\left(U+U^{\dagger}\right)_{12} \\
& -2 r_{0} f_{s}\left(\sqrt{ } U \sigma \sqrt{ } U+\sqrt{ } U^{\dagger} \sigma \sqrt{ } U^{\dagger}\right)_{12}
\end{aligned}
$$

with

$$
\chi=\chi^{\dagger}=m_{\pi}^{2}=2 r_{0} m_{\text {quark }}
$$


Our previous fits to the pseudoscalar propagator determined the following values for the chiral Lagrangian parameters,

$$
\begin{aligned}
f & =0.1066(24) \\
m_{o} & =0.33(2) \\
r_{o} & =1.99(12) .
\end{aligned}
$$

To lowest order, the local isovector scalar density may be expanded in terms of the pion, $\eta^{\prime}$ and sigma fields,

$$
\overline{\Psi_{2}} \Psi_{1}=2 r_{0}\left(\pi^{+} \eta^{\prime}\right)-4 \sqrt{2} r_{0} f_{s}\left(\sigma^{+}\right)
$$

At tree level, only the sigma propagator gives a positive contribution to the scalar propagator

$$
<\overline{\Psi_{2}} \Psi_{1} \quad \overline{\Psi_{1}} \Psi_{2}>=32 r_{0}^{2} f_{s}^{2} P_{\sigma}
$$

where $P_{\sigma}$ is the $a_{0}$ meson propagator. The $\eta^{\prime}-\pi$ term can only contribute via a meson loop. However, the $\eta^{\prime}$ propagator in the loop is the $\eta^{\prime}$ hairpin propagator with a single insertion of the hairpin mass, $m_{0}^{2}$ (see Figure 2). Including only these terms, the scalar propagator is

$$
<\overline{\Psi_{2}} \Psi_{1} \quad \overline{\Psi_{1}} \Psi_{2}>=32 r_{0}^{2} f_{s}^{2} P_{\sigma}+4 r_{0}^{2} B_{\mathrm{hp}}
$$

Here $B_{h p}$ is the hairpin bubble

$$
B_{\mathrm{hp}}=F T\left\{\frac{1}{V T} \sum_{k} \frac{1}{\left((k+p)^{2}+m_{\pi}^{2}\right)} \frac{-m_{0}^{2}}{\left(k^{2}+m_{\pi}^{2}\right)^{2}}\right\}
$$

and FT means the Fourier transform on $p$. As discussed in the Introduction, the negative sign of the $\eta^{\prime}$ propagator in loops is dictated by the quenching and implies that this meson loop makes a negative contribution to the scalar propagator. It should also be noted that the $\eta^{\prime}$ propagator is more infrared singular than the normal valence pion propagator. This has a dramatic effect on the scalar propagator. In the light quark limit, the quenched theory 
predicts that large negative chiral loop effects should dominate the scalar propagator. Our lattice data give convincing evidence for these effects.

A complete analysis of the scalar propagator must go beyond the lowest order terms given above. The chiral Lagrangian predicts couplings between the scalar meson and $\eta^{\prime}$ - $\pi$ states as well as $\eta^{\prime}-\pi$ rescattering interactions generated by the symmetry breaking terms that give mass to the pion. These higher order terms involve $\eta^{\prime}-\pi$ bubble diagrams and can be resummed into a closed form. Since the chiral invariant interactions between the $\sigma$ and $\eta^{\prime}-\pi$ states include derivative couplings, bubbles with derivative couplings at the $\sigma \eta^{\prime} \pi$ vertex must, in principle, also be included. These terms are less infrared singular than the nonderivative terms and are not expected to play a major role in explaining the large quenched effects seen in the data. In the following analysis, we will neglect such derivative coupling terms. The formulas and results given below are for the equal quark mass case; generalizations to the unequal mass case are straightforward.

As is always the case in propagator studies, the presence of excited states in the spectral function complicates the analysis. As we discussed in Section II, in addition to the propagator for local $\bar{\psi} \psi$ operators, we have calculated propagators with a smeared source at one or both ends. In our previous analysis of the pseudoscalar channel [5], a full multi-state fitting procedure was employed. Here we take a somewhat simpler approach which is suggested by features of the data. We model the spectral function in terms of three components: (1) the $\eta^{\prime}-\pi$ state, (2) a ground-state scalar meson $a_{0}$, and (3) an excited-state scalar meson $a_{0}^{*}$. We perform an analysis of the local-local propagator by first using information from the smeared-local and smeared-smeared propagators to remove the excited-state $a_{0}^{*}$ scalar meson component and then fitting to a formula obtained by resumming all repeated bubble graphs involving the $\eta^{\prime}-\pi$ state and the ground-state $a_{0}$ scalar meson, as depicted in Figure 8. To estimate and remove the contribution of the excited $a_{0}^{*}$ from the local propagator, 
we consider first the case with the heaviest quark, $\kappa=.1400$. Within statistics, all of the scalar propagators for smeared and local sources exhibit the same time-dependence beyond $t=3$. In fact, the smeared-local and smeared-smeared propagators are equal, up to an overall factor, at all time slices, as evidenced by the effective mass plot in Figure 1 . For the heavier quark mass values, the $\eta^{\prime}-\pi$ loop is relatively unimportant, and the smeared and local propagators can be analyzed in terms of a ground-state and excited-state scalar meson. The fact that the smeared-local and smeared-smeared propagators, $\Delta_{S L}(t)$ and $\Delta_{S S}(t)$ are proportional and that both give flat effective mass plots indicates that neither propagator has a significant excited-state component. On the other hand, the local-local propagator clearly has a significant $a_{0}^{*}$ component, as seen in the effective mass plot, Figure 4 .

It is straightforward to estimate the $a_{0}^{*}$ contribution by using the (essentially timeindependent) ratio $\Delta_{S L} / \Delta_{S S}$ to determine the excited state component of $\Delta_{L L}$.

$$
\Delta_{L L}(t)-\frac{\Delta_{S L}}{\Delta_{S S}} \Delta_{S L}(t) \sim C_{a_{0} *} \exp \left(-m_{a_{0} *} t\right)
$$

Numerically, the above procedure applied to the $\kappa=.1400$ propagators gives excited state parameters

$$
C_{a_{0}^{*}}=0.275(30), \quad m_{a_{0}^{*}}=1.86(10)
$$

The large mass obtained emphasizes that the excited state contribution is only important at very short time separations. Similar results are obtained for $\kappa=.1405$ and .1410 , with no significant observed dependence on quark mass. With this information, we can separate off the excited state component of the local-local propagator:

$$
\Delta_{L L}(t)=C_{a_{0}^{*}} \exp \left(-m_{a_{0}^{*}} t\right)+\widetilde{\Delta}_{L L}(t)
$$

where $\widetilde{\Delta}_{L L}(t)$ includes contributions from the $\eta^{\prime}-\pi$ state and from the ground state scalar meson, and will be modelled by the bubble sum depicted in Figure 8 . For the lighter quark 
masses, the $\eta^{\prime}-\pi$ state becomes important, and it is more difficult to disentangle the $a_{0}$ and $a_{0}^{*}$ contributions. In the fits to the local-local propagator described in the next section, we have neglected the quark mass dependence of the excited state $a_{0}^{*}$ term and used the estimate (17) for all quark masses.

By resumming the multiple bubble and scalar propagator graphs shown in Figure 8, the full propagator may be written in terms of the one-loop bubble function $B(p)$ defined in equation (2),

$$
\Delta(p)=-4 r_{0}^{2} B^{r e n}+32 r_{0}^{2}\left(f_{s}-f_{s}\left(2 m_{\pi}^{2} / f^{2}\right) B^{r e n}\right)\left(f_{s}-f_{s}\left(2 m_{\pi}^{2} / f^{2}\right) B^{r e n}\right) P_{a 0}
$$

where $f_{s}$ is the coupling of the scalar meson to the $\bar{\psi} \psi$ density, $B^{r e n}(p)$ is the resummed multiple-bubble function,

$$
B^{r e n}=\frac{B(p)}{1+\left(2 m_{\pi}^{2} / f^{2}\right) B}
$$

and $P_{a 0}$ is the resummed $a_{0}$ propagator,

$$
P_{a 0}^{-1}=\hat{p}^{2}+m_{s}^{2}+32\left(f_{s} m_{\pi}^{2} / f^{2}\right)^{2} B^{r e n}
$$

Note that the negative sign associated with the hairpin insertion has been taken outside of the bubble function, i.e. the functions $B(p)$ and $B^{r e n}(p)$ are both positive.

We use the bubble function $B(p)$ defined for the lattice version of the full chiral Lagrangian with the same lattice spacing as the quark lattice. In $(20,21)$, the lattice fourmomentum is defined by $\hat{p} \equiv 2 \sin (p / 2)$ as appropriate for bosonic propagators. If we are to use the pion masses in Table I, which were determined from fitting the t-space pion propagators, then the pion masses used in lattice propagators above are given by $\tilde{m}_{\pi}=2 \sinh \left(m_{\pi} / 2\right)$.

This analysis includes all contributions to the scalar propagator which can be computed by direct $\eta^{\prime}-\pi$ bubble summation and mixing with the $a_{0}$ scalar meson state. We use these expressions in our analysis of the lattice data on the isovector, scalar propagator. 


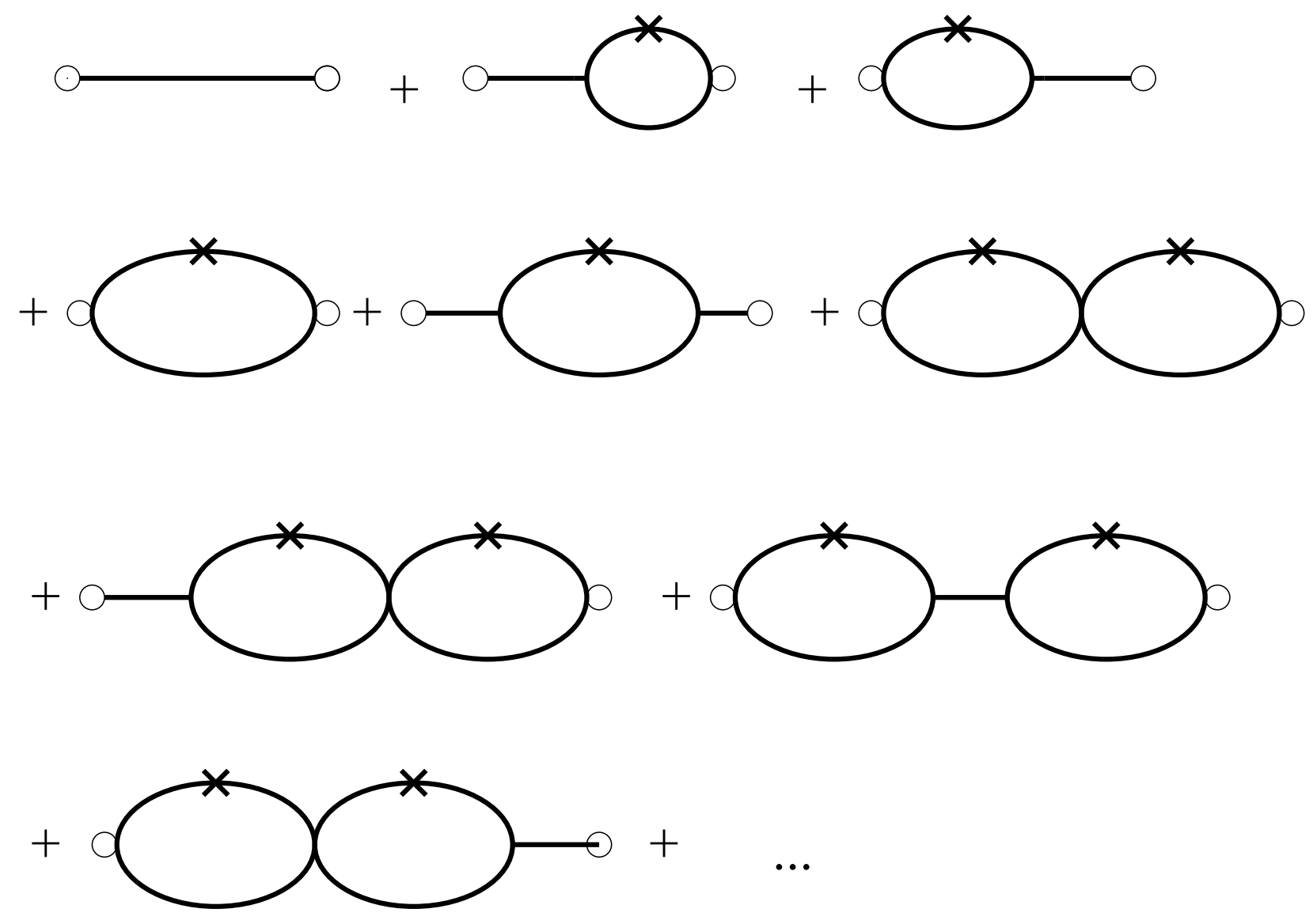

Figure 8: Graphs which are included in the resummed scalar propagator, Eq. (19)) . 
The quenched theory does introduce additional contributions which could modify the behavior of the bubble terms. For example, pions can interact through double hairpin exchange diagrams whose infrared behavior is doubly enhanced. Therefore, virtual $\eta^{\prime}$ processes do not decouple in the infrared limit as is normally expected for soft pion interactions but can generate long range forces between mesons in the quenched theory. Preliminary estimates of these effects indicate they are negligible for the range of pion masses and lattice volume considered in this paper and they have not been included in our analysis.

\section{Global Fits and Determination of Chiral La- grangian Parameters}

In Section 2 we showed that the data for the scalar propagator for heavier quark masses was dominated by the scalar meson $a_{0}$ intermediate state (after removing the excited $a_{0}^{*}$ contribution, as discussed in that Section), while for light masses, it is dominated by the $\eta^{\prime}-\pi$ intermediate state. To get a consistent fit for all masses, we have found that the resummed formula (19) (Figure \&) produces the most stable results. (The simpler alternative of using the sum of a scalar meson pole plus the single bubble function gives fits with only slightly worse $\chi^{2}$ 's but the resulting fit parameters $f_{s}$ and $m_{s}$ are less stable as a function of quark mass.) All of the fits discussed in this section are based on the formula (19) with the parameters $r_{0}$ and $f$ fixed at their previously determined values (11). We present the results of two different fitting procedures. In the first set of fits (Table I), we take the hairpin mass insertion parameter $m_{0}$ to be fixed at the value (11) and use the scalar propagator fits to extract the scalar meson Lagrangian parameters $f_{s}$ and $m_{s}$. In the second set of fits (Table II), we let $m_{0}$ be a fit paramter and investigate how well the scalar propagator data by itself determines the value of the hairpin insertion. 
Table 1: Fit parameters for the scalar propagator, with $m_{0}$ fixed. The fit range is $t=[1-6]$

\begin{tabular}{||c|c|c|c|c||}
\hline$\kappa$ & $m_{P}$ & $f_{s}$ & $m_{s}$ & $\chi^{2} /$ dof \\
\hline .1400 & $.603(2)$ & $.0544(10)$ & $1.20(2)$ & $1.7 / 4$ \\
.1405 & $.556(2)$ & $.0548(10)$ & $1.19(2)$ & $2.0 / 4$ \\
.1410 & $.505(2)$ & $.0550(11)$ & $1.17(2)$ & $1.9 / 4$ \\
.1415 & $.450(3)$ & $.0554(12)$ & $1.16(3)$ & $1.7 / 4$ \\
.1420 & $.386(3)$ & $.0559(14)$ & $1.16(4)$ & $1.7 / 4$ \\
.1423 & $.342(4)$ & $.0565(19)$ & $1.18(5)$ & $2.3 / 4$ \\
.1425 & $.307(4)$ & $.0569(25)$ & $1.19(7)$ & $3.7 / 4$ \\
.1427 & $.267(5)$ & $.0568(38)$ & $1.20(12)$ & $5.6 / 4$ \\
.1428 & $.245(6)$ & $.0571(54)$ & $1.29(18)$ & $6.2 / 4$ \\
\hline
\end{tabular}

The fits which extract the scalar parameters, with $m_{0}$ held fixed, are given in Table 1 . We see that the value of the ground state scalar mass parameter $m_{s}$ is rather well determined for the heaviest quark masses, but becomes less accurate for lighter masses, where the $\eta^{\prime}$ - $\pi$ state dominates. A linear fit to the scalar mass values in Table 1 gives a value of

$$
m_{s}=1.14(7)
$$

in the chiral limit. Using the charmonium scale $a^{-1}=1.18 \mathrm{GeV}$, this gives a scalar meson mass of $1.34(9) \mathrm{GeV}$. The scalar decay constant $f_{s}$ in the chiral limit is

$$
f_{s}=.057(3)
$$

In addition to extracting scalar parameters, it is interesting to carry out fits to the scalar propagator with $m_{0}$ as a fit parameter. This procedure emphasizes the fact that the scalar propagator data alone provides a fairly accurate estimate of $m_{0}$ which is independent of, 
Table 2: Fit parameters for the scalar propagator, with $m_{s}$ fixed to values shown. The fit range is $t=[1-6]$.

\begin{tabular}{||c|c|c|c|c||}
\hline$\kappa$ & $m_{s}$ & $f_{s}$ & $m_{0}$ & $\chi^{2} /$ dof \\
\hline .1400 & 1.200 & $.0568(12)$ & $.336(22)$ & $2.1 / 4$ \\
.1405 & 1.185 & $.0570(12)$ & $.335(25)$ & $2.3 / 4$ \\
.1410 & 1.170 & $.0571(13)$ & $.335(27)$ & $2.3 / 4$ \\
.1415 & 1.156 & $.0574(13)$ & $.338(30)$ & $2.1 / 4$ \\
.1420 & 1.142 & $.0575(14)$ & $.346(33)$ & $2.3 / 4$ \\
.1423 & 1.133 & $.0578(15)$ & $.358(36)$ & $3.0 / 4$ \\
.1425 & 1.128 & $.0576(16)$ & $.360(43)$ & $4.5 / 4$ \\
.1427 & 1.122 & $.0564(18)$ & $.332(51)$ & $6.4 / 4$ \\
.1428 & 1.119 & $.0555(20)$ & $.298(64)$ & $6.7 / 4$ \\
\hline
\end{tabular}

and consistent with, the previous estimates in Reference [5]. As we saw in the previous fits, the scalar mass $m_{s}$ is well-determined for the heaviest quarks, but is poorly determined at the light-quark end. To obtain stable fits with $m_{0}$ as a fit parameter, we fix $m_{s}$ to be given by a linear fit obtained from the three heaviest quark values, $m_{s}=1.106+1.145 m_{q}$. where $m_{q}$ is the bare quark mass $\left(\kappa^{-1}-\kappa_{c}^{-1}\right) / 2$ in lattice units. In this set of fits, $f_{s}$ is found to be approximately independent of quark mass and is well-approximated by (23) for all quark masses. The values of the $\eta^{\prime}$ mass parameter $m_{0}$ obtained from these fits are given in Table 2 and plotted in Figure 9. A linear fit to this data gives

$$
m_{0}=0.34(4)
$$

in the chiral limit. This result, obtained from the scalar propagator data alone, is quite consistent with our previous estimates which included direct $\eta^{\prime}$ hairpin measurements, a topological susceptibility calculation, and QCL effects in the pseudoscalar channel [5]. 


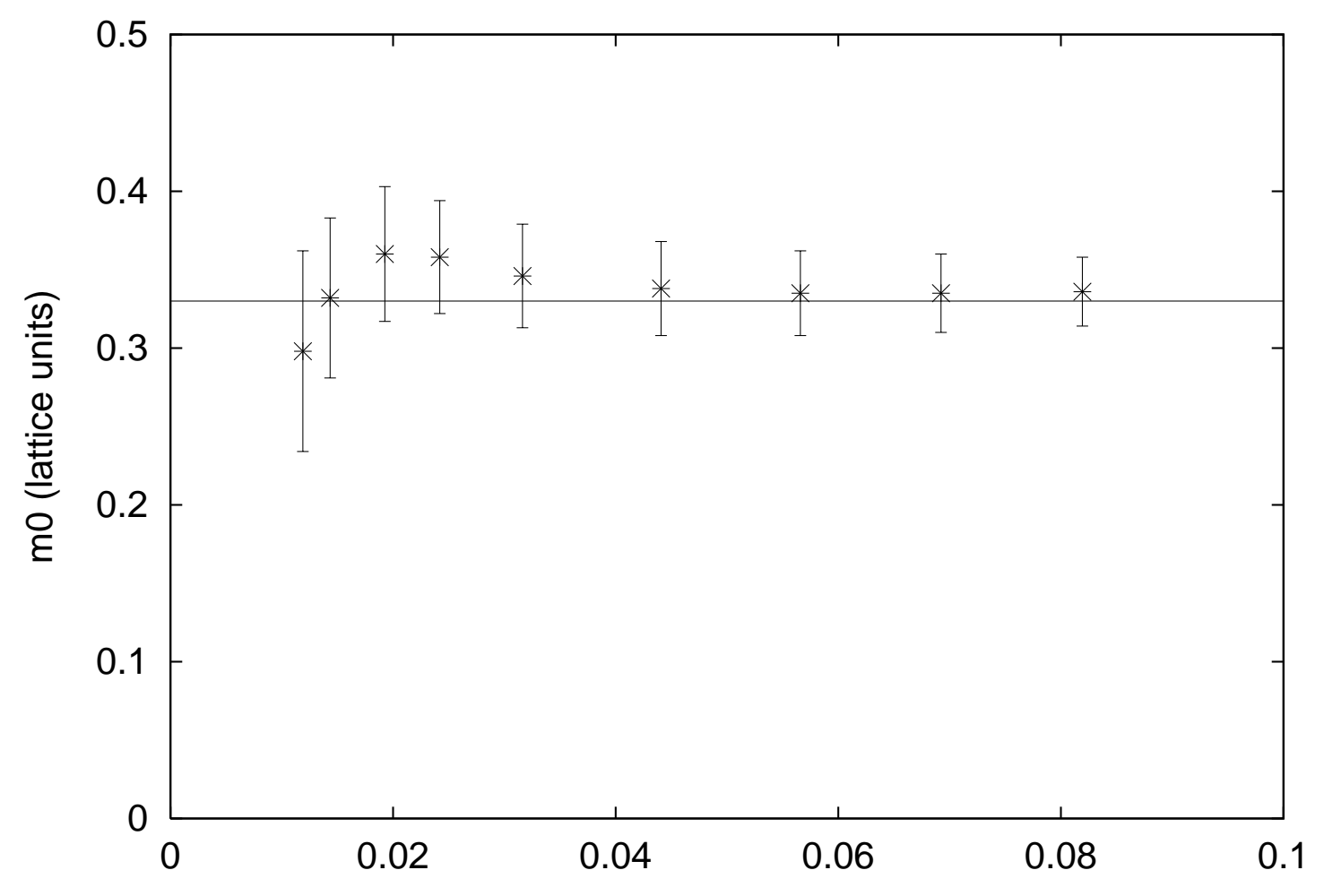

Figure 9: Results for the $\eta^{\prime}$ hairpin mass insertion extracted from fits to the scalar propagator. The solid line is the value obtained from direct analysis of the hairpin diagram in Reference [5] (after removing a $\sqrt{3}$ flavor factor included in the definition of $m_{0}$ in that reference). 
One feature of the scalar propagator which appears to be somewhat inconsistent with our theoretical model is the behavior at larger time separations, $t \geq 7$. For the lightest quarks, we saw that the propagator was well-described in the region $t=3$ to 6 by the oneloop $\eta^{\prime}-\pi$ bubble. However, beyond $t=6$, the propagator appears to go to zero (from below) more rapidly than the theoretical curve predicted by either the one-loop calculation or the bubble-sum formula, as shown for $\kappa=.1427$ in Figure 10. If it is a significant departure from the chiral symmetry prediction, it could be an indication of strong $\eta^{\prime}-\pi$ interactions near threshold. Such interactions would normally be ruled out by soft-pion arguments, but may arise due either to explicit chiral symmetry breaking of the Wilson-Dirac action or because of higher order quenched effects. For the heaviest quarks, we also find that the propagator appears to have an additional positive contribution for $t \geq 7$. For example, we can obtain a good fit (to both the heavy and light mass data at large time) by adding a small $\pi$ propagator term to the fit model. However, the origin of such a contribution is not presently understood within our model.

\section{Discussion}

We have presented convincing evidence that the quenched scalar, isovector meson propagator exhibits a prominent "quenched chiral loop" (QCL) artifact at light quark mass which is properly interpreted as the effect of an $\eta^{\prime}-\pi$ intermediate state. QCL effects that have been observed in other quantities [5, 4] have been rather subtle, involving relatively small deviations from full QCD chiral behavior. By contrast, the QCL effect discussed here is an unmistakable chiral power singularity, and in fact dominates the scalar correlator for the lightest quark masses we have studied. The negative spectral weight of this contribution is a particularly vivid illustration of the oft-stated truism that the quenched approximation 


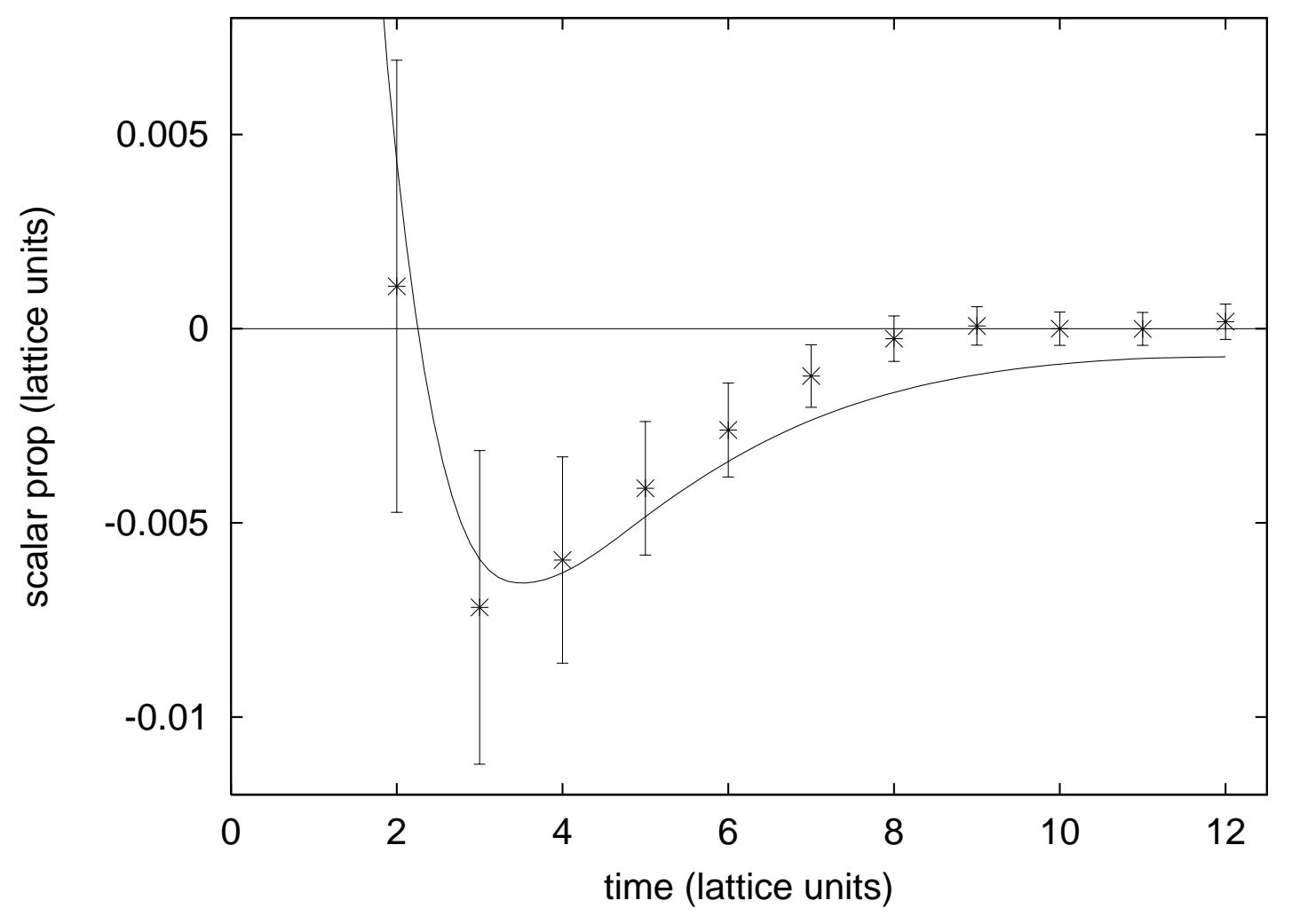

Figure 10: Comparison of scalar propagator for $\kappa=.1427$ with the bubble sum formula (19) ) fitted to the interval $\mathrm{t}=1-6$. 
violates unitarity.

The results presented here also provide further evidence of the effectiveness of the poleshifting ansatz of the modified quenched approximation. It is only after pole-shifting that we are able to study the lightest quark masses for which the $\eta^{\prime}-\pi$ state becomes obvious. It should be remarked, however, that even for the heavier quark masses, the QCL effect is not negligible, and must be included in the fitting function in order to draw correct conclusions about the scalar meson mass. For example, fitting the scalar propagator at the heavier quark masses with a simple exponential and ignoring the $\eta^{\prime}-\pi$ contribution would lead to the erroneous conclusion that the scalar meson becomes heavier as the quark mass gets lighter. The resulting effect on the chiral extrapolation is at least as severe as typical QCL effects in the quenched light hadron spectrum. For pion masses below about 400 $\mathrm{MeV}$, the $\eta^{\prime}-\pi$ contribution becomes dominant, making the scalar meson mass difficult to determine accurately. Nevertheless, by combining lattice calculations with quenched chiral perturbation theory, more extensive studies of scalar meson spectroscopy in the quenched approximation should be feasible. Finally, the prominence of the QCL effect in the scalar valence propagator suggests that this propagator be added to the list of standard bellwether quantities (such as topological susceptibility, $\eta^{\prime}$ mass, and long-range static potential) which are expected to be particularly sensitive to the difference between quenched and full QCD gauge ensembles.

\section{Acknowledgements}

The work of W. Bardeen and E. Eichten was performed at the Fermi National Accelerator Laboratory, which is operated by University Research Association, Inc., under contract DEAC02-76CHO3000. The work of A. Duncan was supported in part by NSF grant PHY00- 
88946. The work of N. Isgur was supported by DOE Contract DE-AC05-84ER40150 under which the Southeastern Universities Research Association (SURA) operates the Thomas Jefferson National Accelerator Facility (Jefferson Lab). The work of H. Thacker was supported in part by the Department of Energy under grant DE-FG02-97ER41027.

\section{References}

[1] S. Sharpe, Phys. Rev. D46 (1992) 3146.

[2] C. Bernard and M. Golterman, Phys. Rev. D46 (1992) 853.

[3] W. Bardeen, et al., Nucl. Phys. B (Proc. Suppl.) 73 (1999) 243.

[4] S. Aoki et al, Phys. Rev. Lett.84 (2000) 238; Nucl. Phys. Proc. Suppl. 73 (1999) 297.

[5] W. Bardeen, A. Duncan, E. Eichten, and H. Thacker, Phys. Rev. D62 (2000) 114505.

[6] W. Bardeen, A. Duncan, E. Eichten, G. Hockney and H. Thacker, Phys. Rev. D57 (1998) 1633. 